\title{
THE EXTREMALS OF TWO INVARIANT INTEGRALS
}

\author{
M. L. MAcQUEEN
}

1. Introduction. In a recent paper, Wilkins shows $[1$, p. 175], 1 among other things, that at a point of an analytic surface in ordinary projective space a general canonical line of the first kind may be characterized in terms of the cusp-axes of the two families of hypergeodesics which are the extremals of a certain pair of invariant integrals.

In this note we introduce two invariant integrals which do not differ essentially from those employed by Wilkins. At each point of the surface, a quadric cone called the cusp-axis cone and its dual called the flex-ray conic are defined in terms of the cusp-axes and flex-rays, respectively, of the hypergeodesics which are the extremals of these integrals. The equations of these loci are derived and some of their properties are briefly studied.

If the four homogeneous projective coordinates $x$ of a variable point on an analytic non-ruled surface $S$ in ordinary space are given as analytic functions of two independent variables $u, v$, and if the parametric net on $S$ is the asymptotic net, then the functions $x$ are solutions of a system of differential equations which may be assumed to be reduced to Fubini's canonical form

(1.1) $x_{u u}=p x+\theta_{u} x_{u}+\beta x_{v}, \quad x_{v v}=q x+\gamma x_{u}+\theta_{v} x_{v} \quad(\theta=\log \beta \gamma)$.

It will be recalled that two lines $l_{1}(a, b), l_{2}(a, b)$ are reciprocal lines $[2, \mathrm{p} .150]$ at a point $x$ of a surface if the line $l_{1}(a, b)$ joins the point $x$ and the point $y$ defined by

$$
y=-a x_{u}-b x_{v}+x_{u v}
$$

and the line $l_{2}(a, b)$ joins the points $\rho, \sigma$ defined by placing

$$
\rho=x_{u}-b x, \quad \sigma=x_{v}-a x,
$$

where $a, b$ are scalar functions of $u, v$.

Moreover, two reciprocal lines $l_{1}(a, b), l_{2}(a, b)$ are canonical lines of the first and second kind respectively in case

$$
a=-k \psi, \quad b=-k \phi,
$$

where $k$ is a constant and

$$
\phi=\left(\log \beta \gamma^{2}\right)_{u}, \quad \psi=\left(\log \beta^{2} \gamma\right)_{v} .
$$

Received by the editors February 8, 1944.

1 Numbers in brackets refer to the references listed at the end of the paper. 
It will be assumed that $\phi \psi \neq 0$, so that the surface under consideration is not a coincidence surface. Canonical lines of the first kind lie in the canonical plane whose local equation is

$$
\phi x_{2}-\psi x_{3}=0,
$$

when referred to the tetrahedron of reference $x, x_{u}, x_{v}, x_{u v}$. The canonical plane intersects the tangent plane $x_{4}=0$ in the first canonical tangent $t_{1}$ at the point $x$ of the surface. Canonical lines of the second kind lie in the tangent plane and pass through the canonical point

$$
(0, \psi,-\phi, 0) \text {. }
$$

The second canonical tangent $t_{2}$, which joins the point $x$ to the canonical point, has the equations

$$
\phi x_{2}+\psi x_{3}=0, \quad x_{4}=0 .
$$

2. The extremals of two invariant integrals. The differential equation of the extremals of the invariant integral

$$
\int_{u_{0}}^{u} \beta^{(2-n) / 3} \gamma^{(1+n) / 3} v^{\prime n} d u \quad(n \neq 0),
$$

where $n$ is a constant, is found to be

$$
v^{\prime \prime}=B_{1} v^{\prime}+C_{1} v^{\prime 2},
$$

in which accents indicate total differentiation with respect to $u$ and the coefficients $B_{1}$ and $C_{1}$ are defined by

(2.3) $3(1-n) B_{1}=\left(\log \beta^{2-n} \gamma^{1+n}\right)_{u},-3 n C_{1}=\left(\log \beta^{2-n} \gamma^{1+n}\right)_{v}$.

These extremals are hypergeodesics whose cusp-axis is the line $l_{1}\left(a_{1}, b_{1}\right)$ for which

$$
\begin{aligned}
& a_{1}=((2 n-1) / 6 n) \psi=-k_{1} \psi, \\
& b_{1}=((2 n-1) / 6(n-1)) \phi=-k_{2} \phi .
\end{aligned}
$$

Similarly, the differential equation of the extremals of the invariant integral

is given by

$$
\int_{u_{0}}^{u} \beta^{(1+n) / 3} \gamma^{(2-n) / 3} \gamma^{1-n} d u \quad(n \neq 1)
$$

$$
v^{\prime \prime}=B_{2} v^{\prime}+C_{2} v^{\prime 2},
$$

where

(2.7) $3 n B_{2}=\left(\log \beta^{1+n} \gamma^{2-n}\right)_{u}, \quad 3(n-1) C_{2}=\left(\log \beta^{1+n} \gamma^{2-n}\right)_{v}$. 
Therefore these extremals are hypergeodesics whose cusp-axis is the line $l_{1}\left(a_{2}, b_{2}\right)$ for which

$$
\begin{aligned}
& a_{2}=((2 n-1) / 6(n-1)) \psi=-k_{2} \psi, \\
& b_{2}=((2 n-1) / 6 n) \phi=-k_{1} \phi .
\end{aligned}
$$

These results can be used to obtain the following theorem, a part of which is similar to Theorem 4.1 in the paper of Wilkins [1, p. 175].

THEOREM 2.1. The extremals of the invariant integrals (2.1), (2.5) are hypergeodesics whose cusp-axes determine the plane

$$
\phi x_{2}+\psi x_{3}-\left((1-2 n)^{2} / 6 n(1-n)\right) \phi \psi x_{4}=0 .
$$

This plane intersects the tangent plane $x_{4}=0$ in the second canonical tangent and intersects the canonical plane in the canonical line $l_{1}(k)$ for which

$$
k=(1-2 n)^{2} / 12 n(1-n)
$$

Moreover, the plane which is determined by the cusp-axis of the hypergeodesics (2.2) and the v-tangent at the point $x$ intersects the plane which is determined by the cusp-axis of the hypergeodesics (2.6) and the u-tangent at the point $x$ in the canonical line $l_{1}(k)$ for which

$$
k=(1-2 n) / 6 n \text {. }
$$

Finally, the cusp-axis of the hypergeodesics (2.2) and the u-tangent at the point $x$ determine a plane which intersects the plane determined by the cusp-axis of the hypergeodesics (2.6) and the v-tangent in the canonical line $l_{1}(k)$ for which

$$
k=(1-2 n) / 6(n-1) .
$$

Each of the canonical lines thus determined may, by a proper selection of the constant $n$, be made to become any desired line of the first canonical pencil, except the first axis of $\check{C}$ ech.

It may be remarked that, in case $n=2$, the two cusp-axes are the scroll directrices of Sullivan. Application of the theorem leads to the canonical line $l_{1}(k)$ for which $k=-3 / 8$, the first edge of Green, and the first directrix of Wilczynski.

3. The cusp-axis cone. We now propose to find the locus of the cusp-axis of the extremals of each of the invariant integrals (2.1), (2.5), when $n$ varies. For this purpose the cusp-axis of the extremals 
of the integral (2.1) can be regarded as determined by the planes whose equations are

$$
x_{3}-k_{2} \phi x_{4}=0, \quad x_{2}-k_{1} \psi x_{4}=0
$$

and the cusp-axis of the extremals of the integral (2.5) by the planes

$$
x_{3}-k_{1} \phi x_{4}=0, \quad x_{2}-k_{2} \psi x_{4}=0,
$$

where $k_{1}$ and $k_{2}$ are defined by

$$
k_{1}=(1-2 n) / 6 n, \quad k_{2}=(1-2 n) / 6(n-1) .
$$

The locus of the cusp-axis (3.1), when $n$ varies, is found by eliminating $n$ from equations (3.1) to be a quadric cone which will be called the cusp-axis cone at the point $x$. The same cone is obtained as the locus of the cusp-axis (3.2). This result can be stated in the following way:

THEOREM 3.1. At a point of a surface, the locus of the cusp-axes of the extremals of the invariant integrals (2.1), (2.5), when $n$ varies, is the cusp-axis cone whose equation is

$$
6 x_{2} x_{3}+\phi x_{2} x_{4}+\psi x_{3} x_{4}=0 .
$$

The vertex of this cone is, of course, the point $x$. It is evident that this cone is intersected by the tangent plane at the point $x$ of the surface in the asymptotic tangents through the point. Moreover, the polar line of the tangent plane $x_{4}=0$ with respect to this cone is the canonical line $l_{1}(k)$ for which $k=-1 / 6$, namely, the first principal line of Fubini and Cech. The canonical plane intersects the cone (3.4) in the projective normal and in the first axis of Cech. The polar plane of the second canonical tangent with respect to the cone is the canonical plane.

It is easy to establish the truth of the following statement:

TheOREM 3.2. The cusp-axes of the extremals (2.2), (2.6) determine the plane (2.9) the polar line of which with respect to the cusp-axis cone is a canonical line $l_{1}(k)$ for which

$$
k=-(1-2 n)^{2} / 6\left(2 n^{2}-2 n+1\right) .
$$

In particular, let us consider the plane determined by the two scroll directrices of Sullivan. The polar line of this plane with respect to the cusp-axis cone is the canonical line $l_{1}(k)$ for which $k=-3 / 10$.

The cusp-axis cone and any quadric of Darboux

$$
x_{2} x_{3}-x_{1} x_{4}+k_{4} x_{4}^{2}=0
$$

( $k_{4}$ arbitrary) 
intersect, besides in the asymptotic tangents at the point $x$, also in a residual conic which lies in the plane whose equation is

$$
x_{1}+\phi x_{2} / 6+\psi x_{3} / 6-k_{4} x_{4}=0 .
$$

This plane cuts the tangent plane at the point $x$ of the surface in the canonical line $l_{2}(k)$ for which $k=-1 / 6$, namely, the reciprocal of the first principal line of Fubini and Cech.

Furthermore, the cusp-axis cone and a principal quadric of Lane [3]

$$
x_{2} x_{3}+x_{4}\left(-3 x_{1}-\phi x_{2} / 2-\psi x_{3} / 2+k_{4} x_{4}\right)=0 \quad\left(k_{4} \text { arbitrary }\right)
$$

intersect, besides in the asymptotic tangents, also in a conic which lies in the plane

$$
x_{1}+2 \phi x_{2} / 9+2 \psi x_{3} / 9-k_{4} x_{4} / 3=0 .
$$

This plane intersects the tangent plane $x_{4}=0$ in the canonical line $l_{2}(k)$ for which $k=-2 / 9$.

4. The flex-ray conic. Let us consider the envelope of the flex-rays of the extremals of the integrals (2.1), (2.5). The flex-ray of the family of hypergeodesics (2.2) is the reciprocal of the cusp-axis (3.1) and has the equations $x_{4}=0$ and

$$
x_{1}+((2 n-1) / 6(n-1)) \phi x_{2}+((2 n-1) / 6 n) \psi x_{3}=0 .
$$

We find that the envelope of this line when $n$ varies is a conic. The same conic is obtained as the envelope of the flex-rays of the hypergeodesics (2.6). This conic will be called the flex-ray conic of the two families of hypergeodesics (2.2), (2.6). The equations of the flex-ray conic can easily be obtained by setting equal to zero the discriminant of equation (4.1) regarded as a quadratic equation in $n$. The result is contained in the following theorem:

TheOREM 4.1. At a point of a surface, the envelope of the flex-rays of the extremals of the two invariant integrals (2.1), (2.5), when $n$ varies, is the flex-ray conic whose equations are $x_{4}=0$ and

$$
x_{1}\left(x_{1}+\phi x_{2} / 3+\psi x_{3} / 3\right)+\left(\phi x_{2}-\psi x_{3}\right)^{2} / 36=0 .
$$

This conic lies in the tangent plane of the surface at the point $x$ and touches the parametric tangents at the point $x$ in the points

$$
(-\phi / 6,1,0,0), \quad(-\psi / 6,0,1,0) \text {. }
$$

The line joining these two points is the reciprocal of the first principal line of Fubini and Cech. This line may therefore be characterized by 
the property that it is the polar line of the point $x$ with respect to the flex-ray conic.

The first canonical tangent intersects the flex-ray conic in the points whose local coordinates are

$$
(-2 \phi \psi / 3, \psi, \phi, 0), \quad(0, \psi, \phi, 0) .
$$

The second axis of Cech is tangent to the flex-ray conic at the first of the points (4.4), and the reciprocal of the projective normal is tangent to the conic at the second of the points (4.4). Thus we find that the canonical point is the pole of the first canonical tangent with respect to the flex-ray conic.

It is easy to verify the following statement:

THEOREM 4.2. The extremals of the two invariant integrals (2.1), (2.5) are hypergeodesics whose flex-rays pass through the point

$$
\left((1-2 n)^{2} / 6 n(1-n)\right) \phi \psi, \psi, \phi, 0
$$

which lies on the first canonical tangent. The harmonic conjugate of the first canonical tangent with respect to the two flex-rays through the point (4.5) is the canonical line $l_{2}(k)$ for which $k$ is defined by (2.10).

The following result is an immediate consequence of the fact that the two flex-rays which pass through the point (4.5) are tangent to the flex-ray conic.

TheOREM 4.3. The polar line of the point (4.5) with respect to the flex-ray conic is the canonical line $l_{2}(k)$ for which $k$ is defined by (3.5).

\section{REFERENCES}

1. J. Ernest Wilkins, The first canonical pencil, Duke Math. J. vol. 10 (1943) pp. 173-178.

2. E. P. Lane, $A$ treatise on projective differential geometry, University of Chicago Press, 1942.

3. - Invariants of intersection of two curves on a surface, Amer. J. Math. vol. 54 (1932) pp. 699-706.

SOUThWESTERN COLLEGE 